\title{
Gender effects in perceived recognition as a physicist and physics identity
}

\author{
Ewan Bottomley ${ }^{1,2}$, Kenneth I. Mavor, and Paula J. Miles \\ ${ }^{1}$ School of Psychology and Neuroscience, University of St Andrews, St Andrews, KY16 9JP, United Kingdom \\ Antje Kohnle and Vivienne Wild \\ ${ }^{2}$ School of Physics and Astronomy, University of St Andrews, St Andrews, KY16 9SS, United Kingdom
}

This study investigated gender differences in perceived recognition as a physicist and its relation to physics identity. We surveyed 688 physics majors ( 228 women, 460 men) at a research-intensive university in the UK at both the lower and upper undergraduate levels with items measuring perceived recognition and physics identity. Similarly to other studies, we find that women report lower recognition as a physicist from their instructors, families and friends compared to men. In contrast, there were no gender differences in students' perceptions of friends asking them for their advice/input in physics-related problems. Perceived recognition from instructors was lower than recognition from family and friends for both men and women. We find that both perceived recognition from instructors and physics identity are significantly lower for upper level students compared with lower level students. Multiple linear regressions for men and women individually found that both perceived recognition from instructors and from family/friends predicted students' physics identity. These results may indicate a shift in students' understanding of what it means to be a physicist as they progress through the degree program. The results point to further research being needed to understand better the mechanism by which students form perceptions of instructors seeing them as physics people. 


\section{INTRODUCTION}

Recently, a number of researchers have examined to what extent students in high school physics and in introductory university calculus-based physics believe they are perceived as physicists by others, and the impact this has upon their academic experience [1-4]. The belief of being recognized as a physicist by others (which we denote as "perceived recognition" in what follows) has positive implications for the formation of a students' physics identity [2]. A students' physics identity is how they view themselves in relation to physics as a discipline. For example, a student with a strong physics identity believes that physics is central to their sense of self. Students who feel that they are recognized as a physicist by others are more likely to see themselves as a physicist [2, 3]. This relationship between perceived recognition and physics identity is important as physics identity has been associated with various positive outcomes such as better academic performance [5] and a greater willingness to continue to study physics in the future [6].

In a study of students taking introductory university calculus-based physics (generally engineering and physical science students), Kalender and colleagues [2] have recently demonstrated that this perceived recognition as a physicist may depend on gender, with women in their study on average reporting lower perceived recognition as a physicist compared to men. They also demonstrated that gender was indirectly associated with physics identity via perceived recognition from others. This is problematic as lower perceived recognition is associated with lower physics identity. Gender in their study was also significantly indirectly associated with competency beliefs and interest in physics through perceived recognition, with women on average having lower interest in physics and lower competency beliefs compared to men. These results indicate that women may have lower perceived recognition as a physicist from others compared with men, and that this can negatively impact their relationship with physics as a discipline.

Studies that have examined perceived recognition as a physicist have operationalized this concept using a survey with items developed by Hazari and colleagues [6]. This twoitem measure is based on Gee's [7] assertion that being recognized as a "kind of person" is influential with regards to the formation of people's identity. These two items are: "My parents/relatives/friends see me as a physics person", and "My teacher see me as a physics person". More recently, a third item has also been used to capture perceived recognition: "Do your friends seek your advice/input in physics-related problems?" (e.g. [8]). This measure gives a general, global indication of the perceived recognition from others in physics students.

There are benefits to using the global version of this scale (averaging across all items to obtain a single score) in terms of understanding how a sense of recognition relates to other variables. In this study, however, we are interested in responses to individual perceived recognition items, their relation to gen- der and physics identity, and whether or not responses vary for lower and upper level undergraduate students. This allows us, for example, to compare students' perceptions of being recognized as a physicist by parents/relatives/friends and by instructors. Previous work has found that high-school teachers' recognition of students was beneficial in enhancing students' physics identity [9] and persistence to continue in physics [10].

Physics identity studies have shown that context matters (e.g. [11]). The nature of students' identity will differ depending on factors such as the group dynamics, environment, and students' beliefs about themselves in relation to their discipline of study. Given the relationship between perceived recognition and identity (e.g. [2, 3]), it seems plausible that perceived recognition could also depend upon context. Hazari and colleagues [11] demonstrated that associations between physics identity and a sense of belonging in women were only present in senior year students, not first year students.

In this work, we investigate gender differences in perceived recognition, and whether such differences vary with level of study and by whom students are being recognized. One could hypothesize that students will feel less recognized by instructors than by parents/relatives/friends, as instructors may have higher expectations of what it means to be a physicist as in-group members of the physics community. One could also hypothesize that perceived recognition by parents/relatives/friends would persist across the levels of the undergraduate degree, whereas perceived recognition by instructors could initially be lower and increase as students progress through the degree program and gain expertise.

This study investigated the following research questions:

RQ1: Are there gender differences in perceived recognition by parents/relatives/friends and instructors, and do they vary from the lower to the upper levels?

RQ2: To what extent do the individual perceived recognition survey items predict physics identity in men and women?

This article is structured as follows: Section II describes the data collection and study details. Section III A relates to RQ1 and Section III B to RQ2. Section IV discusses the results and their implications.

\section{DATA COLLECTION AND METHODS}

The participants in this study were physics students from a small research-intensive institution in the UK. All students majored in a physics degree (e.g., physics, astrophysics, etc.). The students were surveyed across all undergraduate levels, from the introductory level (level 1) to the integrated masters level (level 5). The dataset divided by levels is shown in Table I. Students were given class time to complete the perceived recognition and identity items as part of a larger survey that also sampled other constructs. Students' involvement in the study was voluntary. Data collection was repeated at the start and end of two academic years, and the start of a third aca- 
demic year, i.e., five timepoints in total. Not all levels were surveyed at each of these timepoints, leading to the variation in numbers in Table I. Excepting for one of the timepoints, participation was high, with $60 \%$ to $100 \%$ of students enrolled in a course in which the survey was run completing the survey. There are cases where the same student completed the survey at multiple timepoints. After removing responses with missing data on the core items of interest (29 responses), the total dataset comprised 688 surveys, with 228 from women and 460 from men.

TABLE I. The total number of survey responses and the percentage of men and women at each level.

\begin{tabular}{cccc}
\hline \hline Level & Total & Men & Women \\
\hline 1 & 113 & $70.8 \%$ & $29.2 \%$ \\
2 & 91 & $54.9 \%$ & $45.1 \%$ \\
3 & 309 & $72.5 \%$ & $27.5 \%$ \\
4 & 123 & $61.8 \%$ & $38.2 \%$ \\
5 & 52 & $57.7 \%$ & $42.3 \%$ \\
\hline \hline
\end{tabular}

\section{A. Survey items}

The survey included the adapted version of the three items described above as the measure of perceived recognition. The items used were as follows:

1. Do your parents/relatives/friends see you as a physics person?

2. Do your teachers/instructors see you as a physics person?

3. Do your friends seek your advice/input in physicsrelated problems?

Rather than the standard procedure of averaging the responses of these three items, we chose to treat each item as an individual variable in order to assess differences between them. The survey also examined physics identity, using the single item from Hazari and colleagues' [6] study:

4. I see myself as a physics person.

All items were measured on a 7-point Likert Scale (1=not at all, $7=$ very much so).

\section{RESULTS}

\section{A. Correlations and gender differences in perceived recognition}

Analyses were carried out in SPSS and Mplus. To examine the relationship between the four survey items, we determined the Pearson's correlations between them. These correlations can be seen in Table II. The table shows that the items correlated positively moderately with one another for the overall sample.
TABLE II. The correlations between the items split by gender (228 responses from women, 460 from men), with the correlations for women above the diagonal and the correlations for men below the diagonal. For brevity, the items are only written out in the column header. Starred values indicate $p<.001$.

\begin{tabular}{|c|c|c|c|c|}
\hline Item & 1 & 2 & 3 & 4 \\
\hline $\begin{array}{l}\text { 1. Do your parents/ } \\
\text { relatives/friends } \\
\text { see you as a } \\
\text { physics person? }\end{array}$ & 1 & $.574 *$ & $.449 *$ & $.574 *$ \\
\hline $\begin{array}{l}\text { 2. Do your } \\
\text { teachers/instructors } \\
\text { see you as a } \\
\text { a physics person? }\end{array}$ & $.500 *$ & 1 & $.459 *$ & $.566^{*}$ \\
\hline $\begin{array}{l}\text { 3. Do your friends } \\
\text { seek your advice/ } \\
\text { input in physics- } \\
\text { related problems? }\end{array}$ & $.416^{*}$ & $.417 *$ & 1 & $.447 *$ \\
\hline $\begin{array}{l}\text { 4. I see myself } \\
\text { as a physics person. }\end{array}$ & $.513^{*}$ & $.508 *$ & $.336^{*}$ & 1 \\
\hline
\end{tabular}

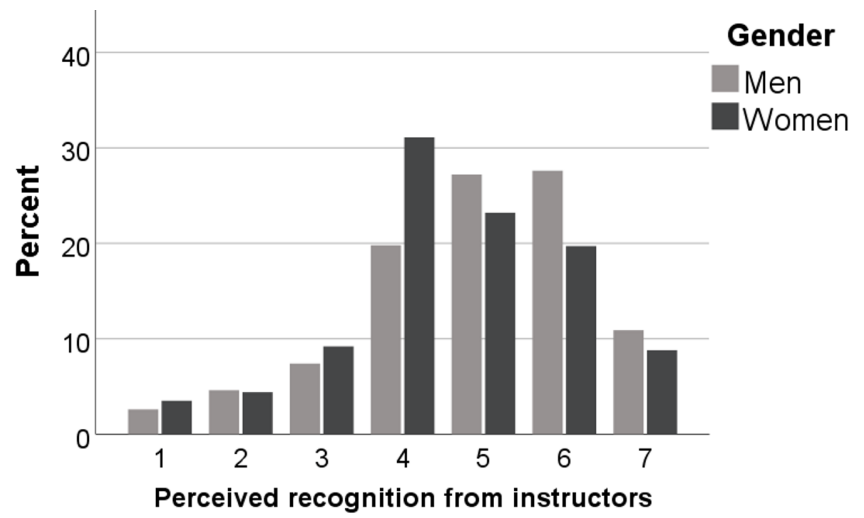

FIG. 1. The Likert Scale responses (1=not at all, $7=$ very much so) in percent for perceived recognition from instructors, separated by gender.

As one example of the data, Fig. 1 shows the Likert-scale responses in percent to the perceived recognition from instructors item. One can see gender differences at the higher end of the scale.

We then examined the gender differences between all four items using a 2 (gender: men vs. women) $\times 2$ (level: lower vs. upper) between-subjects MANOVA. The lower level consisted of those in levels 1 and 2, the upper level consisted of those in levels 3, 4, and 5, where level 5 is the integrated masters level. The means and standard errors for these four items separated by gender and level can be seen in Table III.

In what follows, we cite effect sizes via the partial eta 
squared $\left(\eta_{p}^{2}\right)$. This is the proportion of variance accounted for by an independent variable, controlling for the effect of all other independent variables and interactions on the dependent variable. All effect sizes for the significant differences were small (0.01 to 0.03) but not trivial. Examination of the multivariate tests showed that there was a significant main effect of gender, $F(4,681)=5.188, p<.001$. The pairwise comparisons showed that men reported significantly greater recognition from family and friends than women (the differences between the mean: $M_{\text {diff }}=.458, S E=.129, p<.001$, $\left.\eta_{p}^{2}=.018\right)$. Similarly, men reported greater perceived recognition from their instructors $\left(M_{\text {diff }}=.346, S E=.123\right.$, $p=.005, \eta_{p}^{2}=.011$, see also Fig. 1) and greater physics identity $\left(M_{\text {diff }}=.527, S E=.125, p<.001, \eta_{p}^{2}=.025\right)$, compared to women. However, no significant differences emerged between men and women in terms of the extent to which they felt others sought their advice $\left(M_{\text {diff }}=.164\right.$, $\left.S E=.122, p=.178, \eta_{p}^{2}=.003\right)$.

TABLE III. Means and standard errors on the means (in parentheses) for the four survey items, separated by gender and level. "Lower" refers to levels 1 and 2, "upper" to levels 3, 4, and 5 of the degree program. Numbers of responses were 130 (Men Upper), 330 (Men Lower), 74 (Women Lower), and 154 (Women Upper).

\begin{tabular}{lll}
\hline \hline Item & Gender/Level & Mean $(S E)$ \\
\hline 1. Do your parents/ & Men Lower & $5.80(.13)$ \\
relatives/friends & Men Upper & $5.79(.08)$ \\
see you as a & Women Lower & $5.50(.17)$ \\
physics person? & Women Upper & $5.17(.12)$ \\
\hline 2. Do your & Men Lower & $5.35(.12)$ \\
teachers/instructors & Men Upper & $4.73(.07)$ \\
see you as a & Women Lower & $4.95(.16)$ \\
a physics person? & Women Upper & $4.44(.11)$ \\
\hline 3. Do your friends & Men Lower & $5.34(.12)$ \\
seek your advice/ & Men Upper & $5.31(.08)$ \\
input in physics- & Women Lower & $5.30(.16)$ \\
related problems? & Women Upper & $5.02(.11)$ \\
\hline & Men Lower & $5.47(.13)$ \\
4. I see myself & Men Upper & $5.18(.08)$ \\
as a physics person. & Women Lower & $5.18(.17)$ \\
& Women Upper & $4.42(.12)$ \\
\hline \hline
\end{tabular}

The results also showed a main effect of level, $F(4,681)=$ 7.616, $p<.001$. The pairwise comparisons showed that there were no significant differences between the upper and lower levels in terms of whether their family and friends perceived them as a physicist $\left(M_{\text {diff }}=.173, S E=.129\right.$, $\left.p=.179, \eta_{p}^{2}=.003\right)$ nor the extent to which they felt others sought their advice $\left(M_{\text {diff }}=.155, S E=.122\right.$, $p=.203, \eta_{p}^{2}=.002$ ). Surprisingly, lower level students reported greater perceived recognition from instructors $\left(M_{\text {diff }}=.559, S E=.123, p<.001, \eta_{p}^{2}=.029\right)$ and greater physics identity $\left(M_{\text {diff }}=.527, S E=.125\right.$, $\left.p<.001, \eta_{p}^{2}=.025\right)$, compared to the upper level students. The interaction between gender and level was non-significant, $F(4,681)=1.870, p=.114$.

\section{B. Predicting physics identity}

To see whether the items predicted physics identity for men and women, we ran two multiple regression models, one for the men in the sample and another for the women. For these analyses we examined the students in the first timepoint of data collection to avoid having multiple responses from the same student (103 women and 197 men). The regression model for men significantly predicted physics identity $\left(R^{2}=.403, F(3,193)=43.36, p<.001\right)$. The $R^{2}$ value here indicates that $40.3 \%$ of the variance in physics identity is accounted for by the model. In what follows, $\beta$ is the standardized regression coefficient giving the predicted change in units of standard deviations of physics identity for a one standard deviation change in the predictor (while controlling for the other predictors). Examination of the coefficients suggested that, for men, perceived recognition from family and friends significantly predicted physics identity $(\beta=.288, p<.001)$. Similarly, perceived recognition from instructors predicted physics identity in men $(\beta=.422$, $p<.001)$. However, men's belief that their friends sought their advice did not add to the prediction of their physics identity $(\beta=.025, p=.705)$ controlling for the recognition of family and friends or instructors.

With regards to the regression model for women, the model predicted physics identity $\left(R^{2}=.358, F(3,99)=18.34\right.$, $p<.001)$. As with men, recognition from family and friends $(\beta=.255, p<.010)$ and recognition from instructors $(\beta=.361, p<.001)$ both predicted physics identity for women. However, like for men, feeling that friends sought their advice on physics-related problems did not significantly predict physics identity $(\beta=.127, p=.152$ ) controlling for other forms of recognition. To ensure that the lower power was not the reason for these results, we then replicated these analyses on the collapsed full dataset stratifying the data to account for multiple responses from the same student. This produced a similar pattern of results.

\section{DISCUSSION}

With regards to RQ1, the MANOVA (section III A) found that men felt more recognized as a physicist from their family/friends and from instructors than women. However, there was no significant difference between men and women in the extent to which they felt their friends sought their advice for physics-related problems.

As shown in Table III, mean scores for perceived recognition from instructors were significantly lower than those from parents/relatives/friends. This may be due to the fact that instructors have a higher expectation of what it means to be a 
physicist, or due to students being aware of the gap between their own knowledge and that of the instructors in their interactions with them.

Somewhat surprisingly, physics identity and perceived recognition from instructor scores decreased from the lower to the upper level (see Table III and the MANOVA outcomes). It is worth noting that the lower level students (levels 1 and 2) in this study take classes from other subjects (typically mathematics) as well as physics courses, and that the fraction of physics majors in their physics courses is only $65 \%-85 \%$. In contrast, the upper level students (levels 3 to 5) typically only take physics courses, and the fraction of physics majors is close to $100 \%$. Thus, one possible interpretation could be that students' perceptions of being a physicist could shift from a comparison across groups (e.g., physics majors versus students with other majors) to within groups (only physics majors) from the lower to the upper level. The move to the upper level may also coincide with a shift to a deeper understanding of what it means to be a physicist, making students more aware of the gap between their own knowledge and that of the instructors.

With regards to RQ2, the multiple regression models (section III B) significantly predicted physics identity for men and women from the perceived recognition items, with $40.3 \%$ and $35.8 \%$ of the variance in physics identity accounted for by the models for men and women respectively. Notably, the standardized $\beta$ coefficients for perceived recognition from parents/relatives/friends and from instructors were similar in size for both men and women.

Similarly to previous studies [2, 3], we found gender differences in perceived recognition as a physicist from others. This result is problematic, as prior research has suggested that recognition is important in terms of students' desire to pursue with physics in the future [9]. A lack of per- ceived recognition may thus be contributing to the underrepresentation of women that is prevalent in physics higher education (e.g. [12]).

Our results point to further research being needed to understand better the mechanism by which students form perceptions of instructors seeing them as physics people. It would be useful to investigate whether implicit and explicit forms of recognition by teachers identified at the high school level [1] (having high expectations and providing challenges, formal and informal opportunities for collaboration, recognition opportunities in student-centred activities, encouraging students to persist) are equally relevant at the university level. Given the role that instructors may also play in shaping students' physics identity, this seems an important area for future work.

Similarly, a lack of recognition has been associated, both in the current study and in previous literature [2, 3], with lower physics identity. This is problematic, as our results suggest that women reported lower perceived recognition and physics identity. Prior studies have suggested that a lack of physics identity is associated with a lesser desire to continue on with physics study [6], and also with lower academic performance [5]. As such, our obtained association between recognition and physics identity may disproportionately negatively impact women; however causal investigations would be needed to confirm this.

In summary, our results suggest that perceived recognition as a physicist is a valuable variable to examine when trying to understand physics identity. This is important as a gender disparity in perceived recognition may be contributing to women's under-representation in physics. However, more research is needed in order to understand the reasons for the significant decrease in students' perceived recognition from instructors over time.
[1] Z. Hazari and C. Cass, Towards meaningful physics recognition: What does this recognition actually look like?, Phys. Teach. 56, 442 (2018).

[2] Z. Y. Kalender, E. Marshman, C. D. Schunn, T. J. NokesMalach, and C. Singh, Why female science, technology, engineering, and mathematics majors do not identify with physics: They do not think others see them that way, Phys. Rev. Phys. Educ. Res. 15, 020148 (2019).

[3] Z. Y. Kalender, E. Marshman, C. D. Schunn, T. J. NokesMalach, and C. Singh, Gendered patterns in the construction of physics identity from motivational factors, Phys. Rev. Phys. Educ. Res. 15, 020119 (2019).

[4] Y. Li, K. Whitcomb, and C. Singh, How perception of being recognized or not recognized by instructors as a "physics person" impacts male and female students' self-efficacy and performance, Phys. Teach. 58, 484 (2020).

[5] V. Seyranian, A. Madva, N. Duong, N. Abramzon, Y. Tibbetts, and J. M. Harackiewicz, The longitudinal effects of STEM identity and gender on flourishing and achievement in college physics, International Journal of STEM Education 5, 1 (2018).
[6] Z. Hazari, G. Sonnert, P. M. Sadler, and M.-C. Shanahan, Connecting high school physics experiences, outcome expectations, physics identity, and physics career choice: A gender study, J. Res. Sci. Teach. 47, 978 (2010).

[7] J. P. Gee, Chapter 3: Identity as an analytic lens for research in education, Review of Research in Education 25, 99 (2000).

[8] H. Cheng, G. Potvin, R. Khatri, L. H. Kramer, R. M. Lock, and Z. Hazari, Examining physics identity development through two high school interventions, in Physics Education Research Conference 2018 (2018).

[9] J. Wang and Z. Hazari, Promoting high school students' physics identity through explicit and implicit recognition, Phys. Rev. Phys. Educ. Res. 14, 020111 (2018).

[10] Z. Hazari, E. Brewe, R. M. Goertzen, and T. Hodapp, The importance of high school physics teachers for female students' physics identity and persistence, Phys. Teach. 55, 96 (2017).

[11] Z. Hazari, D. Chari, G. Potvin, and E. Brewe, The context dependence of physics identity: Examining the role of performance/competence, recognition, interest, and sense of belonging for lower and upper female physics undergraduates, J. Res. 
Sci. Teach. 57, 1583 (2020).

[12] R. Skibba, Women in physics, Nature Reviews Physics 1, 298

(2019). 